Research Article

\title{
Simulation and Analysis of Wind Pressure Coefficient of Landslide-Type Long-Span Roof Structure
}

\author{
Bin Rong, ${ }^{1,2}$ Shuhao Yin, ${ }^{1}$ Quankui Wang, ${ }^{3}$ Yanhong Yang, ${ }^{3}$ Jian Qiu, ${ }^{3}$ Changsheng Lin, ${ }^{3}$ \\ and Ruoyu Zhang $\mathbb{1}^{1}$ \\ ${ }^{1}$ Department of Civil Engineering, Tianjin University, Tianjin 300072, China \\ ${ }^{2}$ Key Laboratory of Coast Civil Structure Safety, Ministry of Education, Tianjin University, Tianjin 300072, China \\ ${ }^{3}$ The Fourth Construction Engineering Company Ltd. of China Construction Second Engineering Bureau, Beijing, China
}

Correspondence should be addressed to Ruoyu Zhang; zryu@163.com

Received 11 April 2020; Revised 17 December 2020; Accepted 10 February 2021; Published 24 February 2021

Academic Editor: Li Li

Copyright (c) 2021 Bin Rong et al. This is an open access article distributed under the Creative Commons Attribution License, which permits unrestricted use, distribution, and reproduction in any medium, provided the original work is properly cited.

This article carries out a numerical simulation of a landslide-type long-span roof structure, Harbin Wanda Cultural Industry Complex. The maximum span of the landslide-type roof is $150 \mathrm{~m}$ and the minimum span is $90 \mathrm{~m}$, with a minimum height of $40 \mathrm{~m}$ and a maximum height of $120 \mathrm{~m}$, and the roof area is divided into three different parts. The large eddy simulation (LES) method is used to simulate and record the wind pressure coefficient of the roof. The distribution law and cause of the mean wind pressure coefficient of the roof are firstly analyzed, and the comparison with the existing wind tunnel test data proves the validity of the numerical simulation. Secondly, a qualitative analysis is made on the distribution of root mean square (RMS) fluctuating coefficients. Subsequently, the non-Gaussian characteristics of the roof are briefly discussed, and the peak factor distribution is calculated. Finally, based on the total wind pressure coefficient, a simple evaluation method for judging favorable and unfavorable wind direction angles is proposed, and only the shape of the roof and wind angle need to be known.

\section{Introduction}

Large-span structures are widely used in large public buildings such as airports and stadiums due to their light weight, flexible shape changes, and the ability to provide as much space as possible without inner columns. Today, more beautiful long-span structures with strange shapes are being built. However, the characteristics of low damping and large flexibility make long-span structures more sensitive to wind loads. In 2005, affected by the typhoon, one of the seven PTFE top molds on the roof of Beilun Sports Center in Ningbo, China, was completely destroyed, resulting in serious water leakage in the stadium, causing direct economic losses of more than 4 million. In 2005, New Orleans, USA, many metal pieces on the roof of the dome stadium were blown away by the wind, causing the roof to leak water. Long-span wind damage is already a serious problem.

For the wind characteristics of structures, the wind pressure coefficient is a basic research issue. GB50009-2012 [1] lists the recommended wind pressure coefficient distribution values for 39 types of buildings with regular shapes, including flat roofs and sloped roofs. However, the complex shape clearly indicates the need for wind tunnel testing. The wind pressure distribution law of complexshaped structures is not yet mature, and subsequent wind resistance research will be more difficult. In this regard, scholars have carried out a lot of wind pressure studies on complex-shaped structures. Nakamura et al. [2] conducted an aeroelastic wind tunnel test and response analysis on a large-span open-type roof. The results showed that the mean and fluctuating wind pressure coefficients were between $0.1-0.3$ and $0.2-0.5$, respectively. For the pressure fluctuation, the upper surface in the high-frequency area and the lower surface in the low-frequency area had relatively larger fluctuations than other positions. Uematsu et al. [3] tested the wind load characteristics of gable, troughed, and monosloped roofs and proposed the wind coefficient for the design of the cladding and its immediately supporting structures. Based on the discretizing and synthesizing random flow generation (DSRFG) method and a new subgrid-scale (SGS) 
model, combined with FLUENT software, the wind effects of the large-span complex roof of the new Shenzhen railway station were studied by Lu et al. [4]. The results showed good agreement with the distribution of wind pressure coefficients obtained from wind tunnel tests. Combined with the square hyperbolic parabolic roof, Rizzo and Sepe [5] explored the possibility of defining the equivalent static pressure field to reproduce the dynamic displacement envelope of the cables net. As a result of the greater flexibility of membrane structure roofs, data based on rigid wind tunnel tests often have large errors. Takeda et al. [6] added a correction factor $\mu$ based on the rigid wind resistance theory to consider the above effects through investigation. Kwon et al. [7] measured the wind pressure coefficients of four typical singlespan greenhouses (all-span, three-quarter, peach-span, and single-span) in South Korea based on wind direction, roof slope, and roof curvature radius. Man et al. [8] combined wind tunnel test and numerical simulation to study the effect of wind on the net pressure coefficient and flow field of longspan retractable roof structures under different roof conditions. The LES mentioned in the paper is a very effective numerical simulation tool for studying wind pressure characteristics. Relying on actual engineering, Sun et al. [9] conducted wind tunnel tests on ridge-valley tensile membrane structures and explored the effects of wind direction, vector span ratio, eave height, and terrain roughness on wind pressure distribution. Colliers et al. [10] expounded a prototype method of a hyperbolic thin-shell wind tunnel model with integrated pressure sensors and gave the wind pressure coefficient distribution of a hyperbolic parabolic canopy with a high corner under attack. Chen et al. [11] carried out experimental research on the internal and external pressures of long-span single domes and double adjacent domes and analyzed Gaussian and non-Gaussian crest factors. Regarding the large-span canopy roof, Rizzo et al. [12] discussed its pressure coefficients, peak factor distributions, and non-Gaussian characteristics of the pressure time histories and evaluated the combination factors.

In addition to the wind tunnel test, numerical wind tunnel simulation based on computational fluid dynamics (CFD), especially LES, is of low cost, requires short cycles, and has high efficiency. Full-scale simulation can be performed to simulate real situations as much as possible. Blocken [13] summarized the development of computational wind engineering (CWE) in the past 50 years and took CFD as the future development direction. Huang et al. [14] developed a new type of inflow turbulence generator based on discrete synthetic random flow generation (DSRFG) technology, which can generate a pulsating turbulent flow field that satisfies any given spectral line and can improve the accuracy of the research on fluctuating wind characteristics at the expense of appropriately increasing a certain workload. Daniels et al. [15] studied the peak load and surface pressure fluctuations of high-rise buildings by LES. Fouad et al. [16] used CFD technology to simulate the wind pressure distribution of gables, trusses, and domes and focused on comparing the results of gable structures with European codes and ASCE10. The error is about 7\%, and this error has a greater relationship with the meshing. Wijesooriya et al. [17] comprehensively analyzed the effective use of ELES, which allows users to control the predefined RANS-LES area in the domain, as a substitute for a complete LES model. When the RANS-LES interface is properly configured, it can effectively reduce work costs and ensure accuracy.

Harbin Wanda Cultural Industry Complex, shown in Figure 1, is located in Songbei New District, Harbin, China. The overall structure is a super-long landslide-type large-span structure. The maximum span of the landslide-type roof is $150 \mathrm{~m}$ and the minimum span is $90 \mathrm{~m}$, with a minimum height of $40 \mathrm{~m}$ and a maximum height of $120 \mathrm{~m}$. The roof structure is very strange and belongs to the wind-sensitive structure. At present, no one has studied the wind characteristics of this shape. Therefore, it is necessary to perform wind pressure analysis as a typical complex large-span roof structure. In this paper, a large-scale eddy simulation is used to simulate the wind pressure characteristics of the roof under the atmospheric boundary layer, and the mean wind pressure distribution law under different wind directions and full wind directions is analyzed. Additionally, a qualitative analysis of the RMS fluctuating pressure coefficient reflecting the fluctuation of wind pressure is carried out. The non-Gaussian characteristics of the roof are briefly discussed, and the peak factor distribution is calculated. Finally, based on the total mean wind pressure coefficient standard, a simple and rough method for evaluating favorable and unfavorable wind direction angles is proposed only by the shape and wind direction of the structure.

\section{Numerical Simulation}

2.1. Overview of the Project and Model. Harbin Wanda Cultural Industry Complex is located in Harbin, Heilongjiang Province, China, where the terrain type is $\mathrm{B}$ and basic wind pressure is $0.55 \mathrm{kPa}$ according to $\mathrm{GB}$ 50009-2012 [1]. The main body of the building is a landslide-type large-span structure with a maximum span of $150 \mathrm{~m}$ and a minimum span of $90 \mathrm{~m}$. The top of the slope is $120 \mathrm{~m}$ high and the bottom of the slope is $40 \mathrm{~m}$ high. In addition, a skirt room with a height of about 20 meters is built next to the main structure. Figures 2 and 3 show the plane and elevation dimensions of the structure.

The model is established by ICEM CFD as a full-scale model shown in Figure 4, and the model is appropriately simplified. The podium is reduced to a 20 -meter-high rectangular body, and the eaves of areas $\mathrm{A}$ and $\mathrm{C}$ are smoothed; that is, the local wind pressure at the eaves is not considered. The layout of the measurement points and wind direction angles are shown in Figure 5. The analysis of the roof structure is focused, and it is divided into three areas of $\mathrm{A}, \mathrm{B}$, and $\mathrm{C}$ with a total of 84 measurement points. There are 12 kinds of working conditions, divided every $30^{\circ}$.

2.2. Computational Domain and Meshing. The calculation domain containing the model is a prismatic domain, the size of which is $4200 \mathrm{~m} \times 2500 \mathrm{~m} \times 500 \mathrm{~m}$ (downwind $X \times$ crosswind 


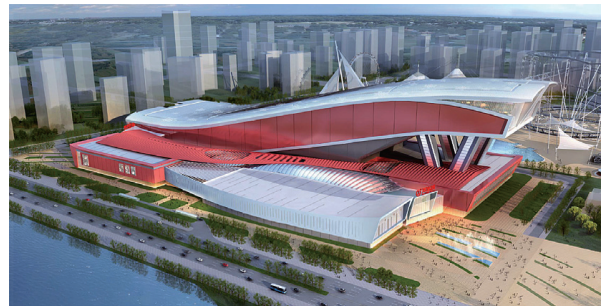

Figure 1: Harbin Wanda Cultural Industry Complex.

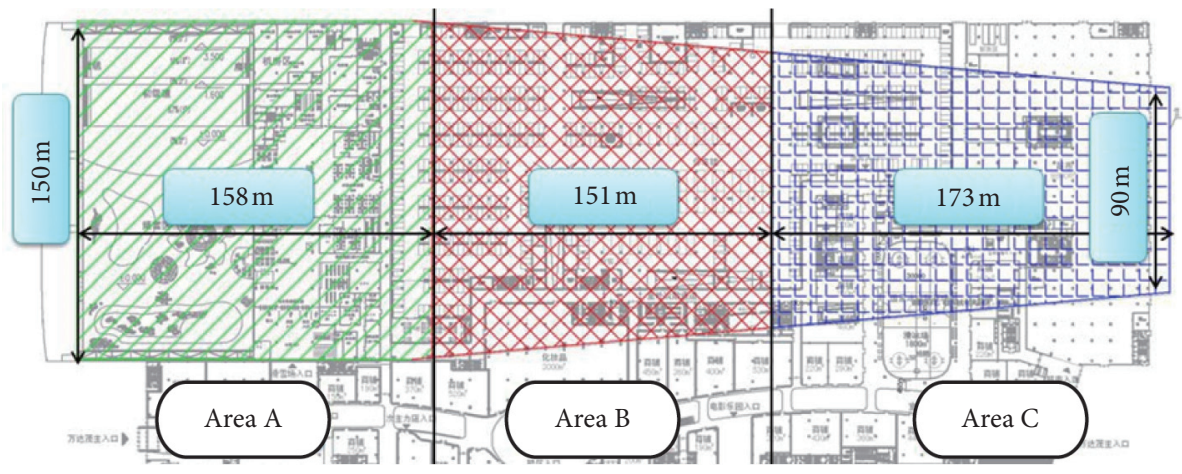

Figure 2: Plane size.

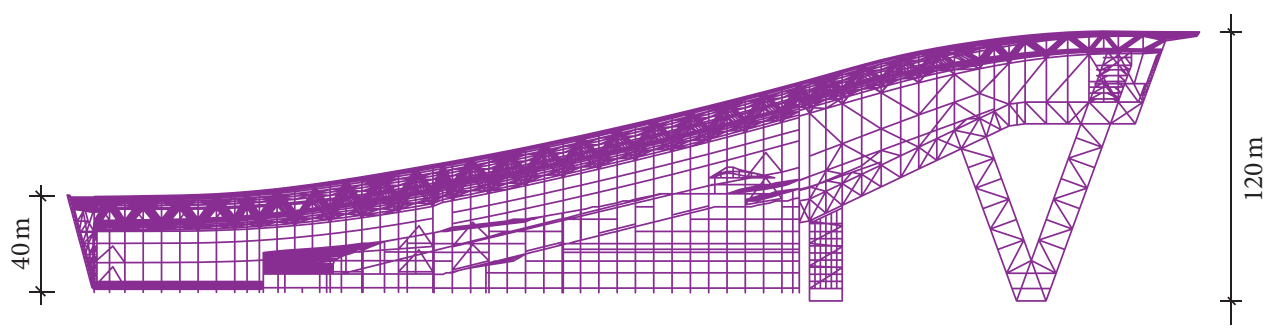

Figure 3: Elevation size.

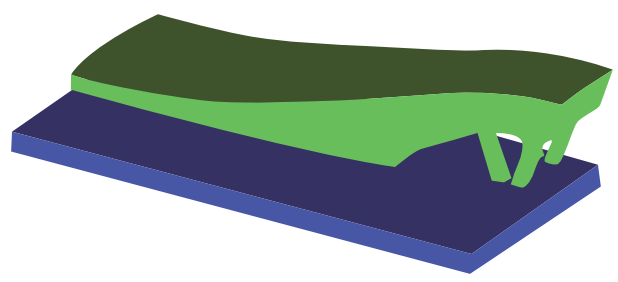

FIgURE 4: Simplified model.

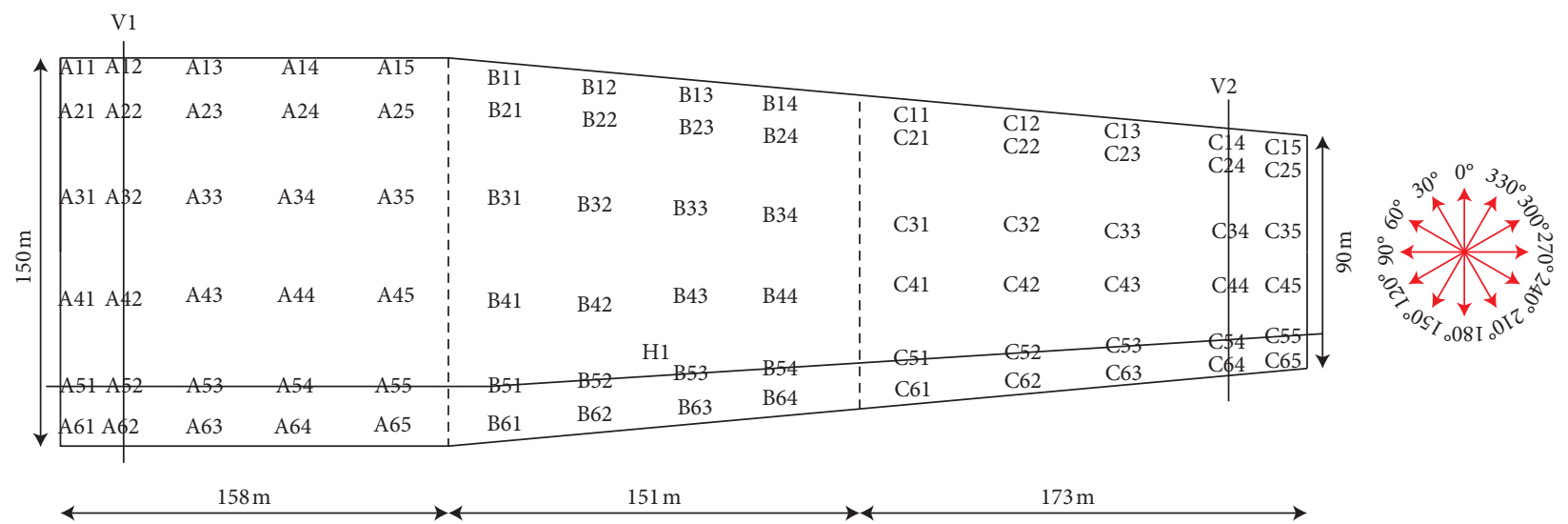

FIgURE 5: Layout of the measurement points and wind direction angles. 
$Y \times$ height direction $\mathrm{Z}$ ). The maximum blockage ratio appears at $0^{\circ}$, which is $2.3 \%$ less than $3 \%$ that is recommended by Franke et al. [18] in the computational wind engineering (CWE) community.

For improving the calculation accuracy and speed, the mesh is divided into two parts: the external area and the internal encryption area including the model. The external area uses high-quality structural hexahedral mesh with a growth factor of 1.05, while unstructured tetrahedral meshes are adopted by internal regions to accommodate irregular shapes. It is worth noting that the LES model is used in the turbulence simulation of high Reynolds number, but the near-wall surface belongs to the low-Reynolds-number region. The turbulent flow development is not sufficient in the near-wall region, and the molecular viscosity plays a leading role. The solution to this problem is to perform mesh encryption on the near-wall area. The size of the first layer of the near-wall area of the model is 0.05 , with a growth factor of 1.1. $y^{+}$calculated by equations (1)-(2) affects the position of the first layer of grid nodes. $y^{+}$needs to be in the logarithm law region (fully developed turbulence region). The total number of grids is $100 \mathrm{~W}$. Element quality is usually used to measure the quality of the grid, and it also affects the convergence of the model. The element quality is between 0 and 1, with 1 being the best. It is generally required that the element quality cannot be negative, and the element quality of this model is above 0.2 . The grid schemes of the $y-z$ plane and the $x-y$ plane are shown in Figures 6 and 7, respectively.

$$
\begin{aligned}
& y^{+}=\frac{y \rho \mu_{\tau}}{\mu}, \\
& \mu_{\tau}=\sqrt{\frac{\tau_{\omega}}{\rho}}
\end{aligned}
$$

where $y$ is the distance between the first layer of mesh node and the wall; $\rho$ is fluid density; $\tau_{\omega}$ is wall shear stress; and $\mu$ is dynamic viscosity.

2.3. Boundary Condition. The structure is simulated under atmospheric boundary conditions for class B terrain. The boundary conditions are as specified in GB50009-2012 [1]. The velocity inlet is the inlet boundary condition. The mean wind speed $V_{Z}$ and turbulence intensity $I_{Z}$ at the inlet are defined by an exponential profile as follows:

$$
\begin{aligned}
& U_{Z}=U_{10}\left(\frac{Z}{10}\right)^{\alpha} \\
& I_{Z}=I_{10}\left(\frac{Z}{10}\right)^{-\alpha}
\end{aligned}
$$

where $U_{10}$ is the maximum mean wind speed during the 50 -year return period of $10 \mathrm{~m}$ in the region, with a value of $29.66 \mathrm{~m} / \mathrm{s} ; I_{10}$ is the nominal turbulence at a height of $10 \mathrm{~m}$, with a value of $0.14 ; Z$ is the distance from the ground to the height; $\alpha$ is the ground roughness index, with a value of 0.15 .

Yan et al. [19] compared several input generation methods of turbulent wind, including the random flow generation (RFG) method, DSRFG method, Vortex method, and Recycling method. Among them, the RFG method modified by Smirnov et al. [20] can better match the target spectrum in the low-frequency (large vortex) interval and requires less workload. This method needs to be provided with turbulent kinetic energy (TKE) and turbulent dissipation rate (TDR), which are defined in CWE as follows:

$$
\begin{aligned}
& \mathrm{TKE}=\frac{3}{2}\left(U_{Z} I_{Z}\right)^{2}, \\
& \mathrm{TDR}=C_{\mu}^{3 / 4} \frac{\mathrm{TKE}^{3 / 4}}{L},
\end{aligned}
$$

where $C_{\mu}$ is a model constant, with a value of $0.09 ; L$ is the turbulent integral length scale, which is suggested by the Japanese standard formula, as follows:

$$
L=100\left(\frac{Z}{30}\right)^{0.5} \text {. }
$$

The pressure outlet is the outlet boundary condition, and relative pressure was set to zero. The bottom surface of the computational domain and the structure surface are treated according to the non-slip-wall surface, and the free-slip-wall surface is applied for the top surface and both sides. The distance from the structure to the entrance is $1400 \mathrm{~m}$. The boundary conditions are set as shown in Figure 8 .

Figures 9 and 10 show the comparison of the simulated values of the boundary conditions with the theoretical values. The simulated values of the mean wind velocity and the turbulence intensity are consistent with the theoretical values. For the power spectral density (PSD), the peak value agrees well with the target spectrum, while the spectrum decays rapidly in the high-frequency range (inertial subrange). Huang et al. [14] believed that the sample spectrum of the RFG method is Gaussian-shaped in the FLUENT code. Considering that the LES method is to solve the large eddy (low frequency) directly, the subgrid model is applied for the small vortex (high-frequency), and the fundamental frequency of the structure is low; it can be considered that the spectrum meets the subsequent simulation requirements to a certain extent.

2.4. Solution Strategy. LES needs to choose the subgrid model to react to the effect of small eddy on large eddy. Dynamic Smagorinsky-Lilly model is applied as the subgrid model, with the model constants $C_{s}=0.1$. The governing equation is solved by pressure-based segregated algorithm in the simulation, where the incoming wind is an incompressible fluid. The pressure-velocity coupling method in the present simulation is PISO (pressure implicit with splitting of operation) algorithm. The Green-Gauss cell-based method is used for numerical approximation of the gradient. The pressure is discretized using the standard scheme. Bounded central differencing (BCD) is used for momentum discretization.

Generally, the average time for wind speed recording is 10 minutes [21]. The cases under the wind direction angles of $120^{\circ}$ were simulated at first, with a time step of $0.05 \mathrm{~s}$ and 


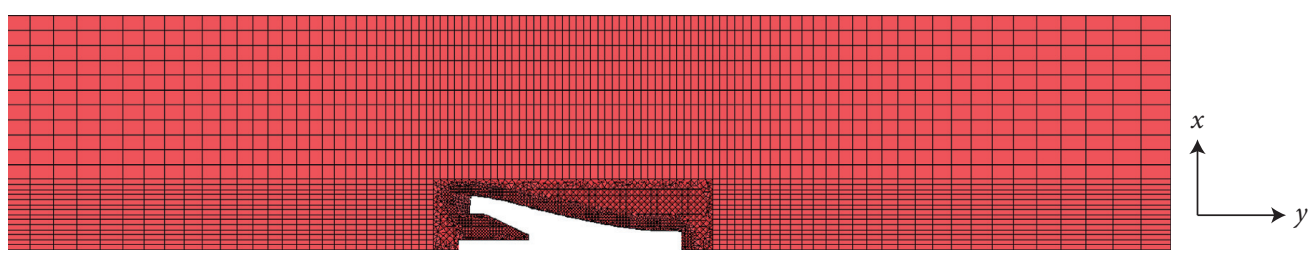

FIgURE 6: Grid scheme on y-z plane.

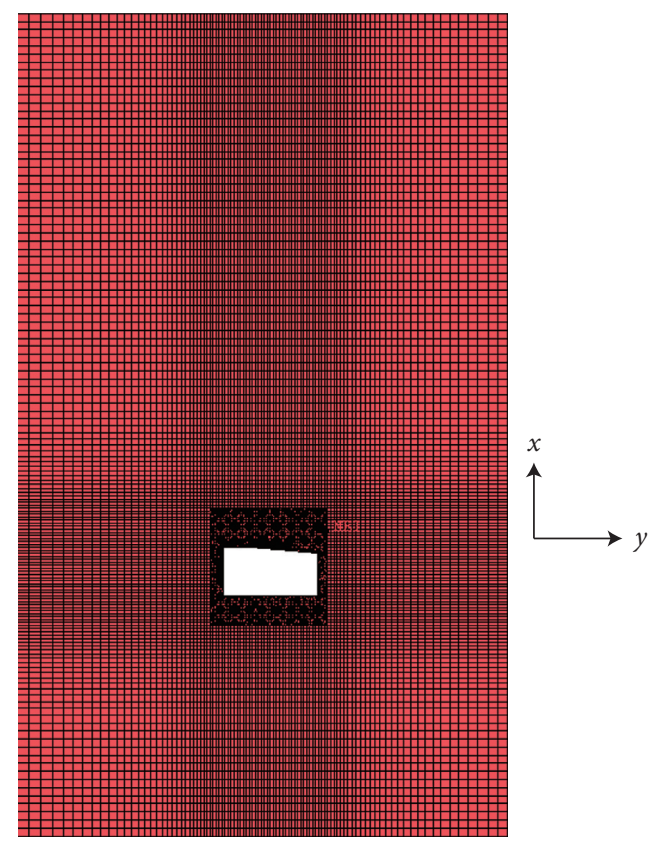

Figure 7: Grid scheme on x-y plane.

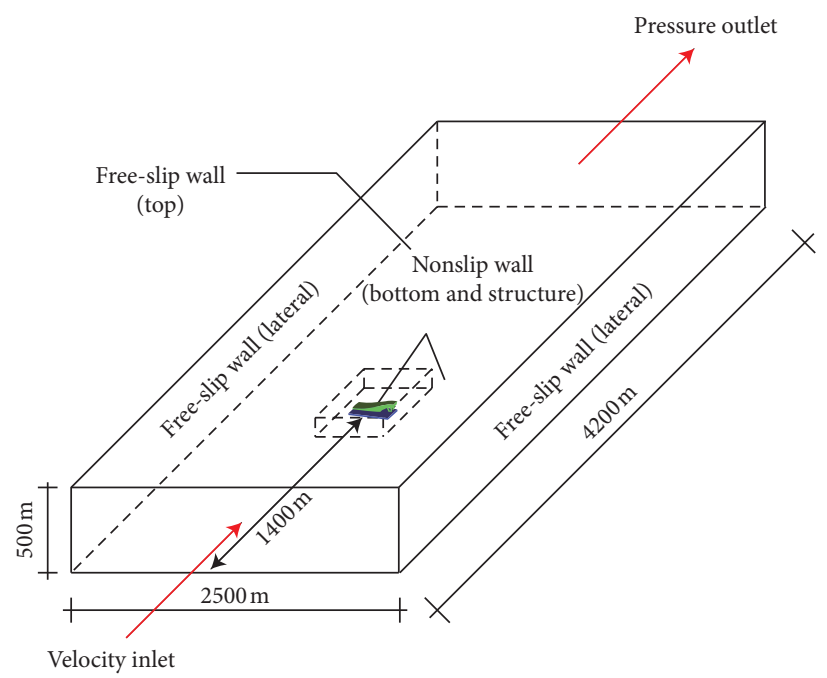

FIgURE 8: Boundary conditions. 


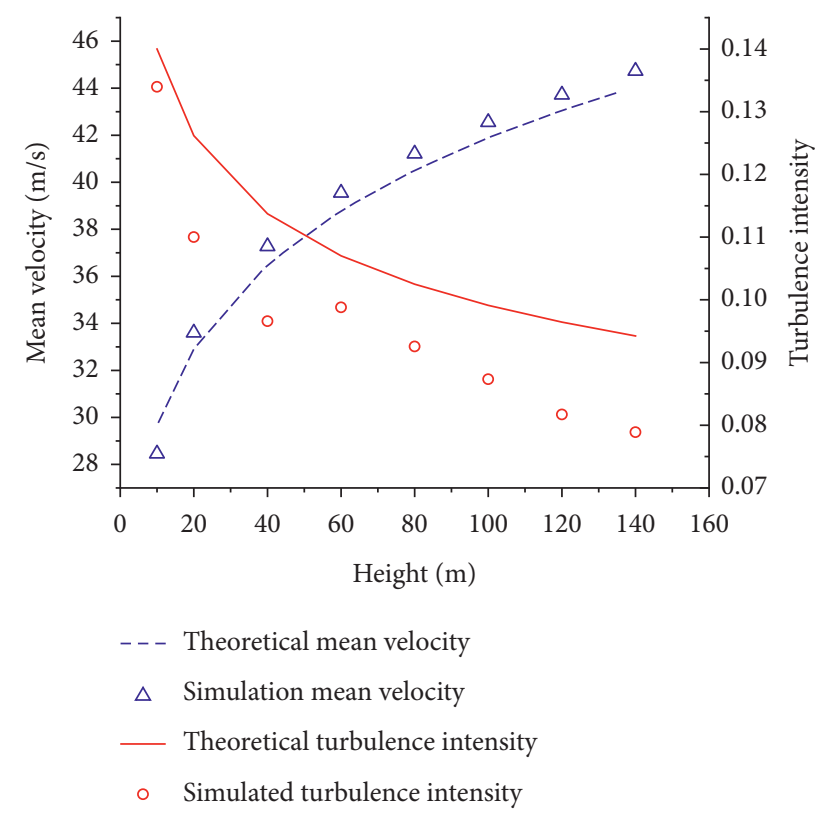

FIGURE 9: Validation of mean velocity and turbulence intensity.

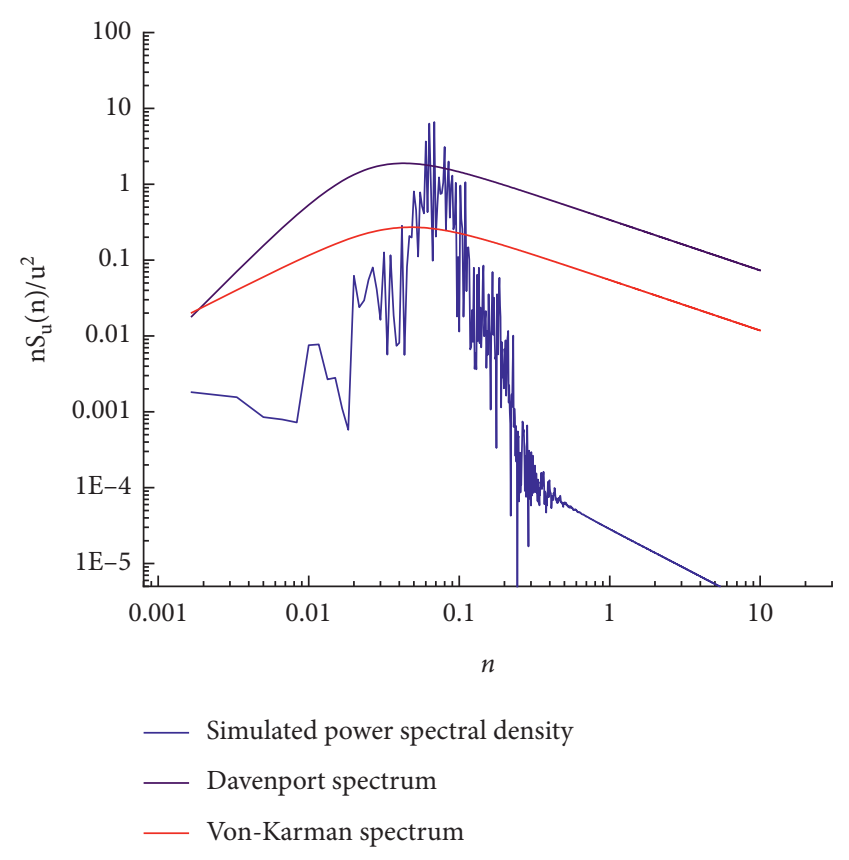

FIgURE 10: Validation of power spectral density.

12000 time steps (600s flow time). The results showed that the time history of wind pressure coefficient of the point C21 in Figure 11 was divided into two parts: invalid domain of the first $150 \mathrm{~s}$ due to the insufficient flow and valid domain of $150 \mathrm{~s}-600 \mathrm{~s}$. For the valid domain, the average values of the wind pressure coefficient of $150-350 \mathrm{~s}$ and $350 \mathrm{~s}-600 \mathrm{~s}$ are -2.91 and -2.92 , respectively, while the fluctuating wind pressure coefficients are 0.6 and 0.7 , respectively. The total of $350 \mathrm{~s}$ flow times are calculated for subsequent cases to obtain converged results and the statistics of the last of $200 \mathrm{~s}$ are sampled for analysis.

\section{Verification and Analysis of Wind Pressure Coefficient}

China Academy of Building Research and CABR Technology Co., Ltd. has completed a wind tunnel test of the Harbin Wanda Cultural Industry Complex. In this chapter, the mean wind pressure coefficient of the roof part of the structure is verified via the comparison of the wind tunnel test and the FE simulation.

The mean pressure coefficient $\overline{C_{p}}$ and the fluctuating pressure coefficient $\widehat{C_{p}}$ at location $i$ are defined as

$$
\begin{aligned}
& \bar{C}_{p}(i)=\frac{\bar{p}(i)-p_{\infty}}{1 / 2 \rho v_{\infty}^{2}}, \\
& \widehat{C_{p}}(i)=\frac{\sigma_{p}(i)}{1 / 2 \rho v_{\infty}^{2}},
\end{aligned}
$$

where $\bar{p}(i)$ is the mean static pressure of $i$ measuring point; $p_{\infty}$ is the static pressure at the infinity; $\rho$ is the density of the air; $\sigma_{p}(i)$ is the root mean square of the static pressure at $i$ measuring point; $v_{\infty}$ is considered here to be the reference wind speed at $10 \mathrm{~m}$ height, for comparison with existing wind tunnel test data.

3.1. Mean Wind Pressure Coefficient. Figures 12(a)-12(f) show the verification results of the mean pressure coefficient under the wind direction of $0^{\circ}, 90^{\circ}$, and $270^{\circ}$, respectively. The wind pressure distribution shows a high similarity. In its entirety, the mean pressure coefficient of the roof is basically negative, which means that it is lower than atmospheric pressure. The separation of eddies at the edges of the roof occurs after the wind contacts the structure, leading to the wind flow being away from the roof in the normal component of the roof and vortex will be generated between the wind flow and the roof. Soon afterwards, wind will attach to the roof away from the contact location, which will cause a slight positive pressure. In addition, the contour line tends to move parallel to the windward edge due to the generation of cylindrical vortices when the wind direction is perpendicular to the windward edge.

For $0^{\circ}$, maximum mean negative pressure occurs at the junction of the windward edge of areas $\mathrm{B}$ and $\mathrm{C}$ and overall shows higher negative pressure. For $90^{\circ}$ and $270^{\circ}$, the FE simulation of the wind pressure distribution shows symmetry. It proves that, under these two wind direction angles, the incidental low building on the side has little effect on the wind pressure of the main structure roof. The reason for the slightly different test results is that the actual wind direction angle of the wind tunnel test is that the wind direction angle specified in this paper has rotated $2^{\circ}-3^{\circ}$ in the instantaneous needle direction. Therefore, the maximum mean negative pressure area of the wind tunnel test is concentrated in the lower-left corner for $90^{\circ}$ and the lower-right corner for $270^{\circ}$. While the FE results about the maximum mean negative pressure appear near the windward edge and form a high negative pressure zone similar to a rectangular area, on the facade, the roof is shaped as a folded landslide as shown in 


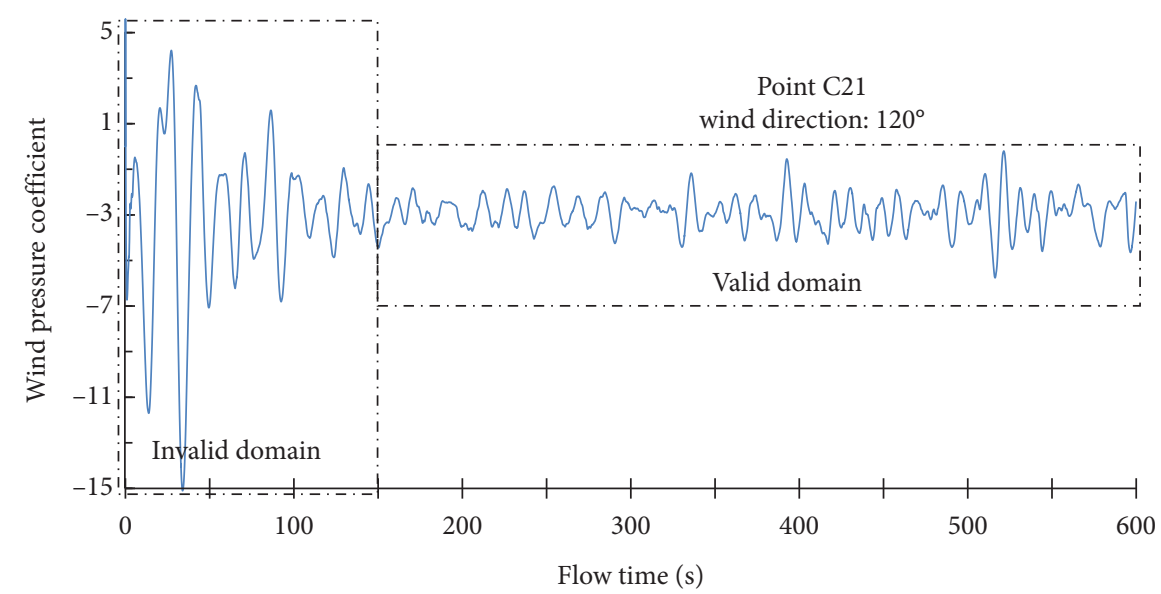

FIgURE 11: Wind pressure coefficient of point C21.

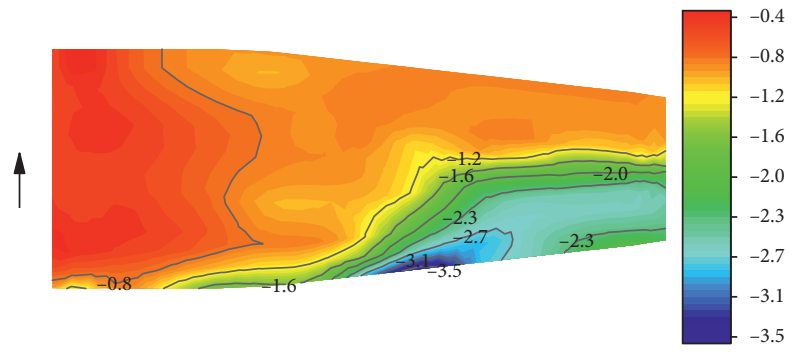

(a)

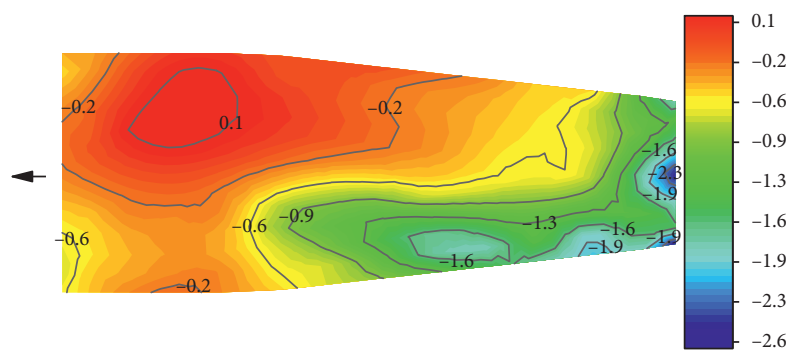

(c)

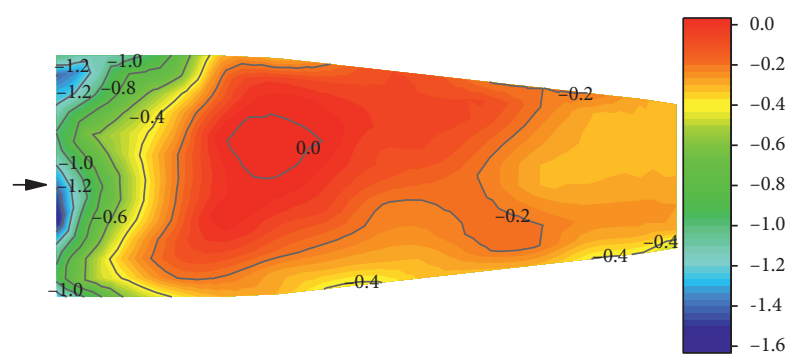

(e)

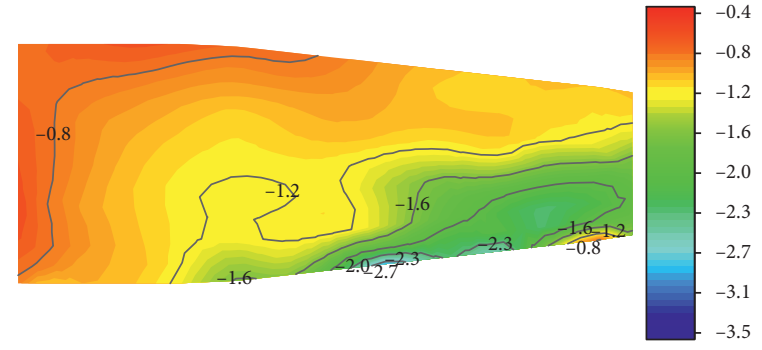

(b)

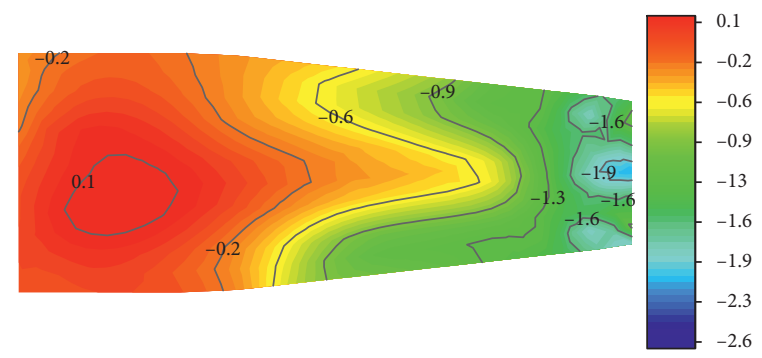

(d)

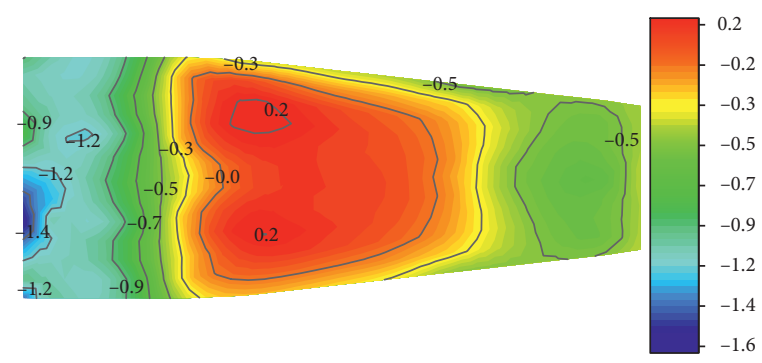

(f)

Figure 12: Continued. 


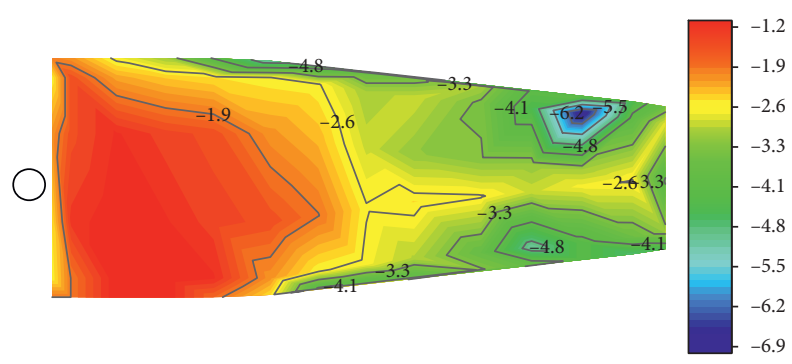

(g)

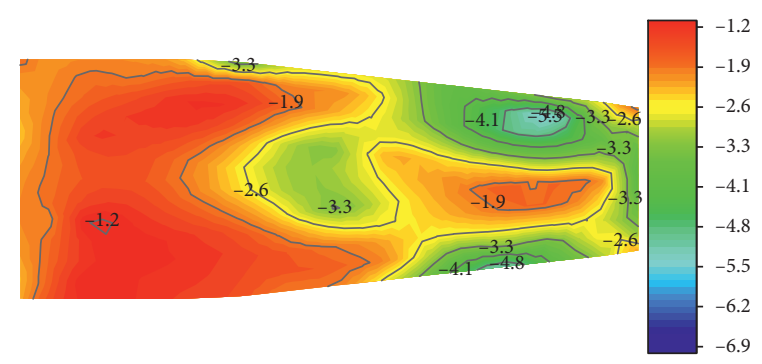

(h)

FIgURE 12: Contour maps of mean wind pressure coefficients for wind tunnel tests and FE simulation. (a) $0^{\circ}$, test, (b) $0^{\circ}, \mathrm{FE},(\mathrm{c}) 90^{\circ}$, test, (d) $90^{\circ}, \mathrm{FE},(\mathrm{e}) 270^{\circ}$, test, (f) $270^{\circ}, \mathrm{FE}$, (g) all directions, test, and (h) all directions, FE.

the simplified Figure 13 . At $90^{\circ}$, the entire surface of area $\mathrm{C}$ is subjected to a large negative pressure. The roof height of areas $\mathrm{A}$ and $\mathrm{B}$ is lower than that of area $\mathrm{C}$, and parallel wind is blocked by buildings in area $\mathrm{C}$, resulting in less wind pressure. At $270^{\circ}$, high negative pressure collects in area A. The parallel wind flows to the surface of the roof in area B and generates a component that is perpendicular to the roof, so a large area of positive pressure appears. When the wind flows to area $\mathrm{C}$, the negative pressure band is reformed, but the negative pressure is relatively small due to the weakening effect of area $B$ on the wind flow.

Figures $12(\mathrm{~g})-12(\mathrm{~h})$ show the statistical minimum value of the mean pressure coefficient at the full wind direction. The results of the wind tunnel test are in the range of -1.2 to -6.9 , while the FE results are within -1.2 to -5.2 . From the perspective of wind pressure distribution, the entire roof maintains a high negative pressure. Relatively speaking, the maximum negative pressure appeared near the junction of the windward edge of area $\mathrm{B}$ and area $\mathrm{C}$, and the overall negative pressure in the middle of area $\mathrm{C}$ and area $\mathrm{A}$ was relatively small. The surface of area $A$ is similar to a square, and the height and width are relatively small, so that the mean wind pressure coefficient of the roof surface does not differ much and remains at a low level. The structure of area $\mathrm{B}$ and area $\mathrm{C}$ is slope-shaped, and the interaction between the upstream vortex and the downstream vortex generated at the windward edge makes the situation complicated, and, finally, a large wind negative pressure is formed near the windward edge of area B and area C.

Table 1 compares the mean wind pressure coefficients of the FE and test data at $0^{\circ}, 90^{\circ}$, and $270^{\circ}$ through two error evaluation methods. The first method is cumulative error method of measuring points. The mean errors at $0^{\circ}$ and 90 are all below $25 \%$ while reaching $38.62 \%$ at $270^{\circ}$. Some measuring points are relatively small (for example, b33, -0.05 ), and the FE results are about -0.1 at the corresponding position. Due to the small error base, although the error value is not large, the error still reaches $100 \%$, which magnifies the overall error. The second method is cumulative error method of total wind pressure. This method ignores the influence of the small value error in Method 1 but cannot reflect the error fluctuation of the measuring point area. The mean errors at $0^{\circ}$ and 90 are all below 25\% while reaching $31.77 \%$ at $270^{\circ}$. When the overall mean pressure coefficient is

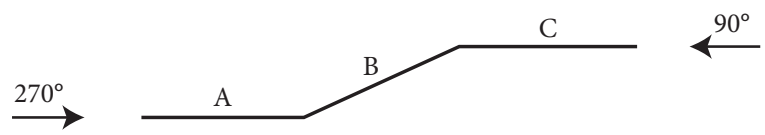

Figure 13: Simplified sketch of the roof at $90^{\circ}$ and $270^{\circ}$ wind direction.

small, this method will produce large errors. For example, the coefficient error value is 0.1 , and the error of the overall mean pressure coefficient value of 1.0 is much smaller than the error of the overall value of 0.3 . The principle of this shortcoming is similar to that in Method 1 to convert small points into overall small values. Based on the above analysis, it can be considered that the FE data and the test data are in good agreement to a certain extent.

The position of the lines of $\mathrm{V} 1, \mathrm{H} 1$, and $\mathrm{V} 2$ can better reflect the change law of the mean wind pressure coefficient under the wind direction condition of $0^{\circ}$. From the comparison between the $\mathrm{FE}$ and the wind tunnel test results shown in Figure 14, the basic distribution trend of the mean wind pressure coefficient is basically consistent. For V1 and $\mathrm{V} 2$, the negative pressure gradually increases from top to bottom, while abrupt changes to a large value occur near the edge of the windward. In fact, the roof surface has a certain arc so that line V2 is an arch. The height of the point C64 is lower than the other points and it is too close to the roof edge, making the wind pressure coefficient slightly smaller than the C54 point. This suggests that negative pressure may not become greater closer to the edge of the windward. The maximum value appears slightly farther from the edge of the roof. For $\mathrm{H} 1$, the negative pressure tends to increase first and then decreases from area $\mathrm{A}$ to area $\mathrm{C}$, and the maximum mean negative pressure appears at the junction of area $B$ and area $\mathrm{C}$. Because of the windward edges of areas $\mathrm{B}$ and $\mathrm{C}$ not perpendicular to the wind flow, a conical vortex is generated above the windward edges, which will cause a relatively large negative pressure in this area. In addition, the slope of the windward edge of area $B$ will increase the turbulence of wind flow, which may also lead to an increase in negative pressure.

This section qualitatively analyzes the distribution law of the mean wind pressure coefficient of the roof at three special wind direction angles $\left(0^{\circ}, 90^{\circ}\right.$, and $\left.270^{\circ}\right)$ and focuses on the law on the three lines (V1, H1, and V2) under $0^{\circ}$ wind direction. The results show that the data obtained by FE 
TABLE 1: Relative error of the mean pressure coefficient.

\begin{tabular}{|c|c|c|c|c|}
\hline & & $0^{\circ}$ & $90^{\circ}$ & $270^{\circ}$ \\
\hline \multirow{2}{*}{ Cumulative error method of measuring points area } & Mean error: $\overline{\delta_{1}}=\sum_{i=1}^{n}|| C_{p i}^{F} / C_{p i}^{T}|-1 \cdot A(i) / A|$ & $23.08 \%$ & $19.28 \%$ & $38.62 \%$ \\
\hline & Variance: $\sigma=\sqrt{\sum_{i=1}^{n}\left[\left(|| C_{p i}^{F} / C_{p i}^{T}|-1|-\overline{\delta_{1}}\right)^{2} \cdot A(i) / A\right]}$ & 0.19 & 0.28 & 0.38 \\
\hline Cumulative error method of total wind pressure & Mean error: $\overline{\delta_{2}}=\sum_{i=1}^{n}\left|C_{p i}^{F}-C_{p i}^{T}\right| \cdot \frac{A(i)}{A} / \sum_{i=1}^{n}\left|C_{p i}^{T} \cdot A(i) / A\right|$, & $19.48 \%$ & $24.94 \%$ & $31.77 \%$ \\
\hline
\end{tabular}

$C_{p i}^{F}$ and $C_{p i}^{T}$ represent mean wind pressure coefficients of measuring point $i$ in FE and test, respectively; for $A(i), A$ is the horizontal projection value of the measured area to the $i$ point and the total horizontal projected area, respectively.

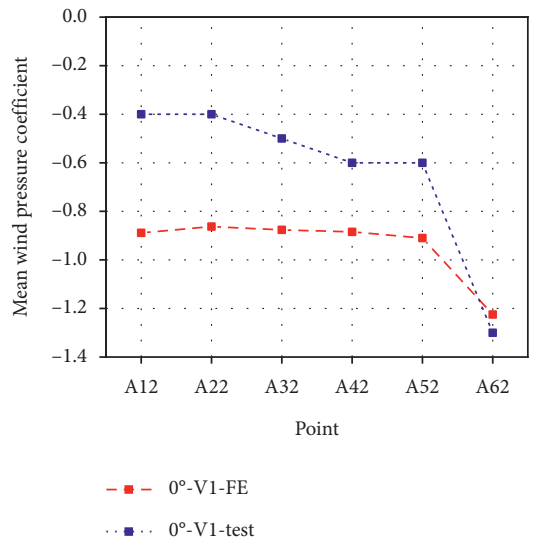

(a)

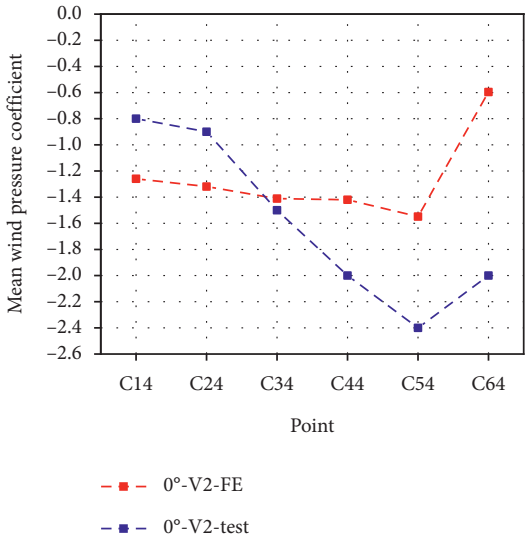

(b)

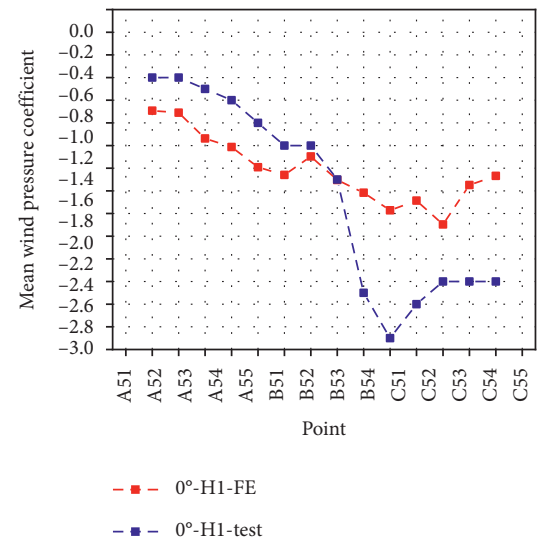

(c)

Figure 14: Comparison of mean wind pressure coefficient between FE and test for $0^{\circ}$. (a) For line V1, (b) for line V2, and (c) for line H1.

method agree well with the distribution law of mean wind pressure coefficient obtained from wind tunnel tests, and the LES simulation results are feasible.

3.2. RMS Fluctuating Pressure Coefficient. In the atmospheric boundary layer, wind turbulence and complex phenomena such as separation and reattachment of wind flow at the windward edge can cause the fluctuation of the wind pressure coefficient on the building surface with time. The turbulence characteristic of the wind pressure coefficient is expressed by the root mean square (RMS) fluctuating pressures, which is an important index in the wind resistance design of the structure. Figure 15 shows the RMS fluctuating pressure coefficient of the roof under the conditions of $0^{\circ}$, $90^{\circ}$, and $270^{\circ}$ and the whole wind direction.

It can be found that the distributions of $0^{\circ}, 90^{\circ}$, and $270^{\circ}$ are similar, all of which are larger near the windward edge and gradually decrease with the distance from the windward edge. The maximum RMS value at $0^{\circ}$ appears at the junction of $B$ and $C$, as a result of the slopes here. Since area A is low, the turbulence intensity is higher here, which is why the high negative pressure fluctuation mainly appears in area A for the RMS fluctuating pressure coefficient in the whole wind direction. These phenomena prove that RMS has a great relationship with the turbulence intensity of the wind. Large RMS values will be generated at low places and complex shape which are prone to high turbulence. Lacking wind tunnel test data of the RMS fluctuating pressure coefficient, only the results of the FE are analyzed. Combining the degree of coincidence of the wind speed spectrum and the mean wind pressure coefficient, this result only conducts a qualitative analysis of the distribution form.

3.3. Non-Gaussian Processes Distribution on the Roof. Table 2 summarizes the skewness and excessive kurtosis maximum, minimum, and mean values for $\theta=0^{\circ}, 90^{\circ}$, and $270^{\circ}$ and for all the pressure taps of the roof. The skewness for $0^{\circ}$ is mainly positive, while $90^{\circ}$ and $270^{\circ}$ are opposite, with values between -0.39 and 0.42 . The excessive kurtosis value is between -0.69 and 0.56. Suresh Kumar and Stathopoulos [22] divided the roof regions of low buildings where pressure coefficients are non-Gaussian processes with the following criteria: $\left|\gamma_{c p}\right|>0.5$ and/or $\left|k_{c p}\right|>0.5$, where $\gamma_{c p}$ is the skewness and $k_{c p}$ is the excessive kurtosis of the process. However, different from the roof of low buildings, the roof in this paper is located at a higher position and is less affected by atmospheric boundary layer turbulence. Based on FE data, a particular region is considered non-Gaussian (colored region) if the absolute values of skewness and excessive kurtosis of pressure fluctuations at various taps are $>0.2$ and $>0.4$, respectively.

In Figure 16, orange regions indicate that $\left|\gamma_{c p}\right|>0.2$, red regions indicate that $\left|k_{c p}\right|>0.4$, and white regions correspond to a Gaussian behavior. We can observe that most of the roof performs Gaussian behavior, with a small amount of non-Gaussian areas concentrated near the windward edge, roof corners, and area B. Basically, the non-Gaussian 


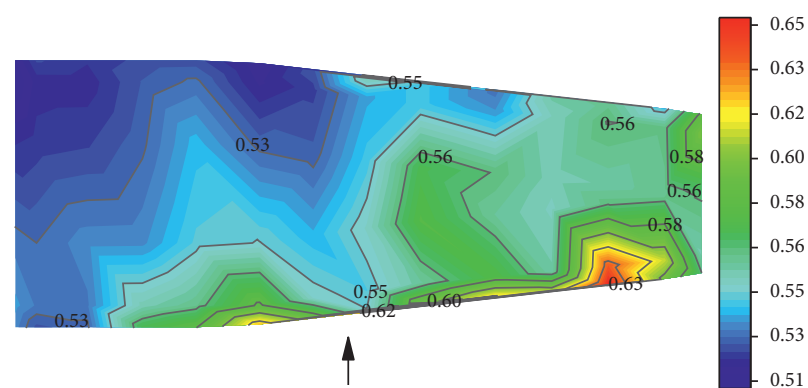

(a)

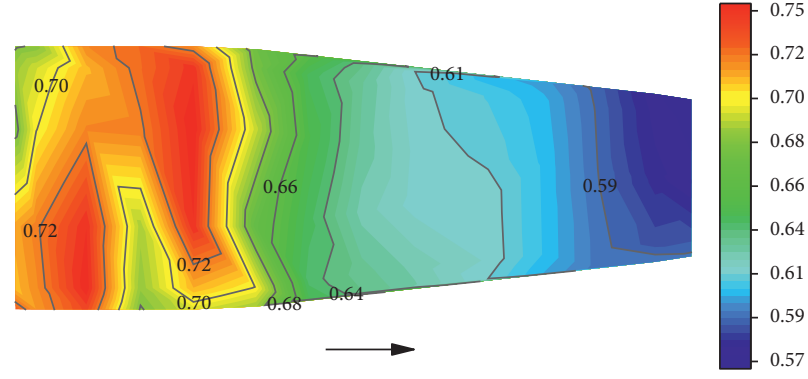

(c)

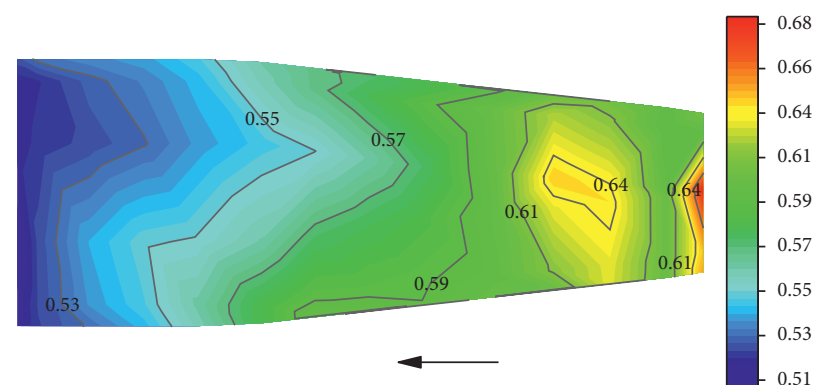

(b)

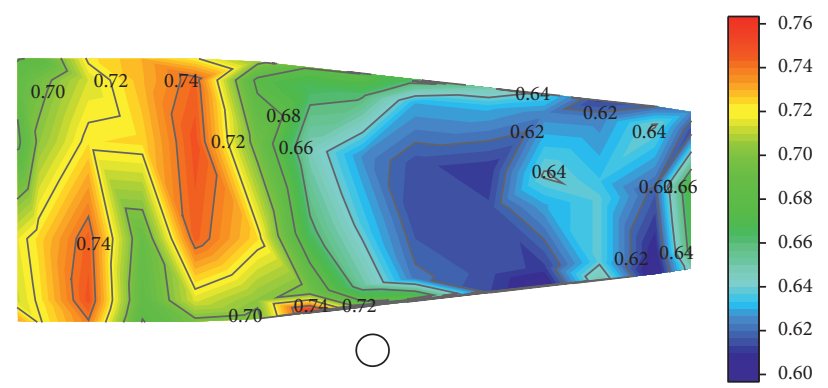

(d)

FIGURE 15: Contour maps of RMS fluctuating pressures for FE simulation. (a) For $0^{\circ}$, (b) for $90^{\circ}$ (c) for $270^{\circ}$, and (d) for whole.

TABLE 2: Skewness and excessive kurtosis of pressure coefficient.

\begin{tabular}{|c|c|c|c|c|c|c|c|c|c|}
\hline & & $0^{\circ}$ & & & $90^{\circ}$ & & & $270^{\circ}$ & \\
\hline & Max & Min & Mean & Max & Min & Mean & Max & Min & Mean \\
\hline$\gamma_{c p}$ & 0.42 & -0.26 & 0.05 & 0.02 & -0.39 & -0.18 & 0.06 & -0.37 & -0.15 \\
\hline$k_{c p}$ & 0.56 & -0.53 & -0.11 & 0.28 & -0.69 & -0.27 & 0.27 & -0.43 & -0.20 \\
\hline
\end{tabular}

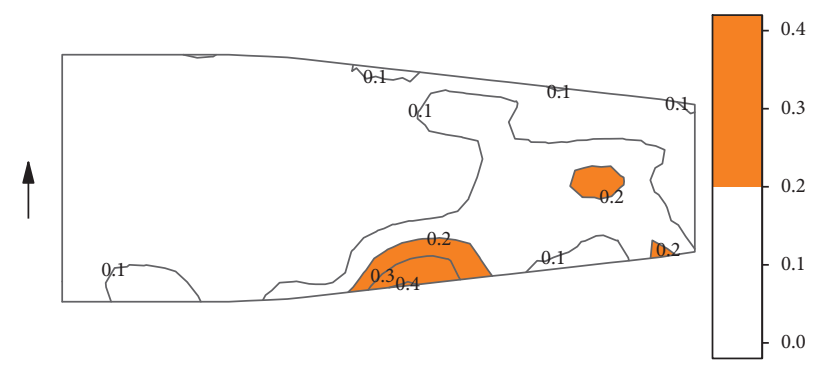

(a)

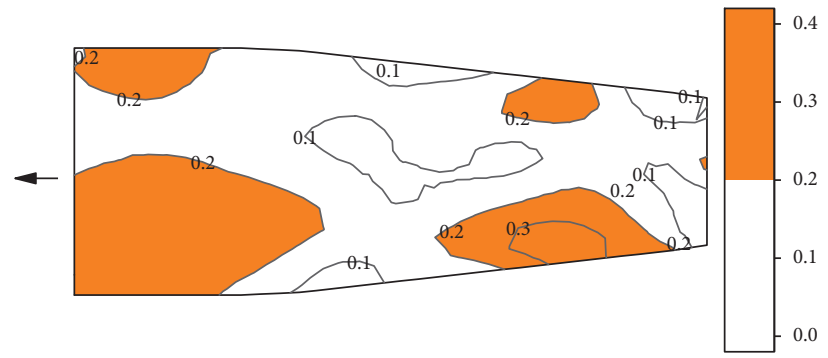

(c)

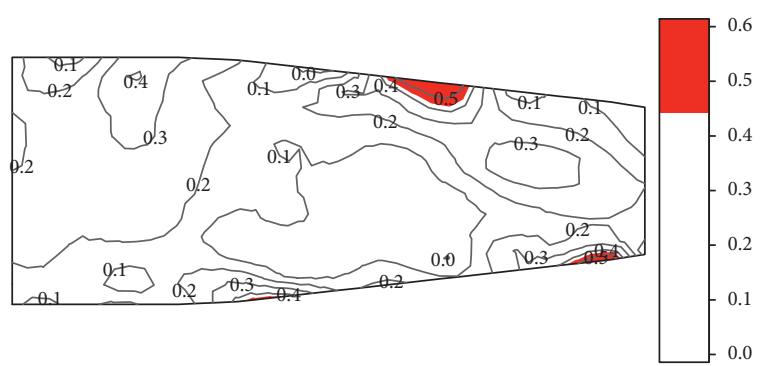

(b)

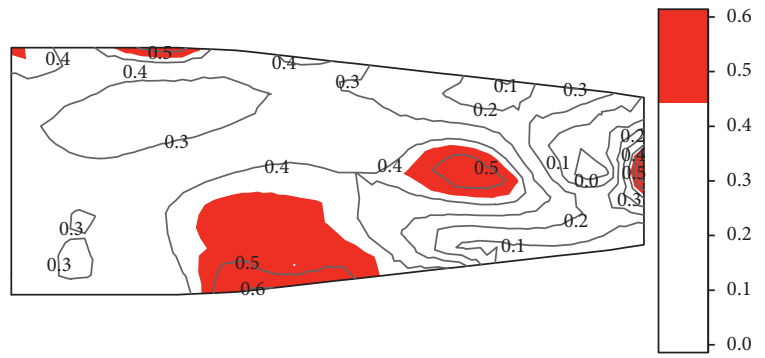

(d)

FIgURE 16: Continued. 


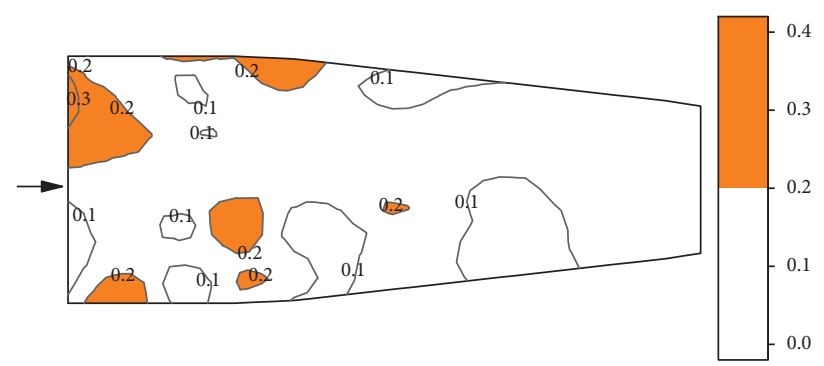

(e)

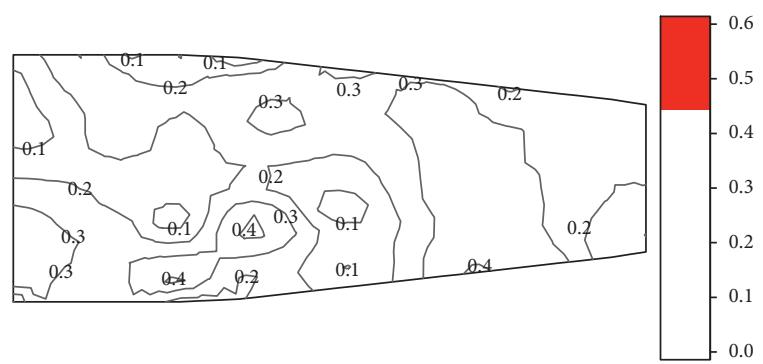

(f)

Figure 16: Gaussian and non-Gaussian processes: (a) skewness for $0^{\circ}$, (b) kurtosis for $0^{\circ}$, (c) skewness for $90^{\circ}$, (d) kurtosis for $90^{\circ}$, (e) skewness for $270^{\circ}$, and (f) kurtosis for $270^{\circ}$. Orange regions correspond to $\left|\gamma_{c p}\right|>0.2$; red regions correspond to $\left|k_{c p}\right|>0.4$; white regions correspond to Gaussian behavior.

behavior is not very serious as a result of the high position of the roof with an insignificant effect of the turbulence of the atmospheric boundary layer.

3.4. Pressure Peak Factors. In structural design, stability analysis requires accurate extreme wind loads, which are determined by the extreme wind pressure coefficient. The extreme wind pressure coefficient is usually determined by equations (7)-(8), taking into account the peak factors (maximum $-g_{\max }$ and minimum $-g_{\min }$ ), as follows:

$$
\begin{aligned}
& C_{p, \text { max }}=\overline{C_{p}}+g_{\max } \sigma_{C p}, \\
& C_{p, \min }=\overline{C_{p}}-g_{\min } \sigma_{C p},
\end{aligned}
$$

where $C_{p, \max }$ and $C_{p, \min }$ represent the maxima and the minima pressure coefficients based on the data of FE; $\overline{C_{p}}$ and $\sigma_{C p}$ represent the mean and the standard deviation of the pressure coefficient recorded by the measure taps.

Figures 17(a)-17(f) show the distribution of the peak factors of the pressure for wind angles of $0^{\circ}, 90^{\circ}$, and $270^{\circ}$, respectively. We can observe that the peak factors are all less than 3.5, which is the design value commonly adopted by wind loading codes. Specifically, the maximum peak factor at $0^{\circ}$ is 3.5, larger than the other two angles. The maximum peak factor of all angles appears near the windward edge, and the change gradient of the peak factor at this position is larger, which is caused by the more complicated changes in the incoming flow at the windward edge.

\section{Discussion of the Unfavorable Wind Direction}

When studying the wind load characteristics of structures with complex shapes, it is often necessary to analyze the operating conditions from many wind directions, and we often only need to analyze some unfavorable ones. Adverse working conditions also need to be determined according to the required research content. In this chapter, we try to discuss the unfavorable wind direction based on the total wind pressure, which is of great significance in grasping the overall wind load in wind resistance design. Additionally, the most unfavorable total air volume research method has been transformed through data analysis, which can greatly reduce the workload of determining unfavorable working conditions. Meanwhile the method is rough, which is only suitable for preliminary rough judgment.

4.1. Analysis of Total Wind Pressure Coefficient Standard. The total wind pressure is an overall evaluation of the wind pressure on the building surface. We can define a representative value of the coefficient to consider the impact of the overall average wind pressure as follows:

$$
\overline{C_{p t}}=\sum_{i=1}^{n} \frac{\left|\overline{C_{p}(i)}\right| A(i)}{A}
$$

where $A(i)$ is the horizontal projection value of the measured area corresponding to the $i$ point; $A$ is the total horizontal projected area.

In order to simplify the calculation, $A(i)$ and $A$ are considered as the horizontal projected area. The error can be maintained within a controllable range due to the fact that the curvature of the roof surface is small and the slope angle of area $B$ is within $15^{\circ}$. The wind pressure on the surface of the roof is composed of most of the negative pressure and a small part of the positive pressure. The mean wind pressure coefficient of the measurement point $i$ takes the absolute value to consider the influence of the total wind pressure. The total mean wind pressure coefficient of the roof at each wind direction and the dividing line is shown in Figure 18. The roof is symmetrical along the $90^{\circ}$ and $270^{\circ}$ directions. It can be analyzed that the data at the two ends of the dividing line are basically symmetrical, while there is a slight error due to the influence of the podium. The maximum total mean wind pressure coefficient appears at $150^{\circ}$, reaching 1.5 , which can be considered the most unfavorable situation of the total wind pressure. By the way, all operating conditions except $90^{\circ}, 240^{\circ}, 270^{\circ}$, and $300^{\circ}$ are maintained at a total mean wind pressure coefficient value above 1 and similar. To a certain extent, these are all unfavorable working conditions of negative pressure. In contrast, for this type of roof, we can conclude that the favorable working conditions of negative pressure are $90^{\circ}$ and $270^{\circ}$. Structural engineers can consider 


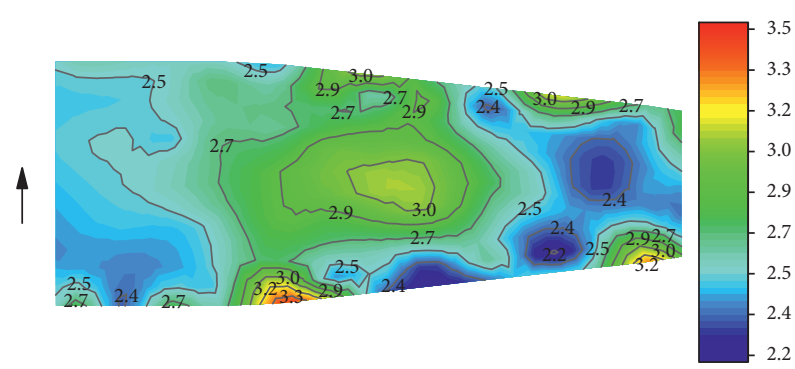

(a)

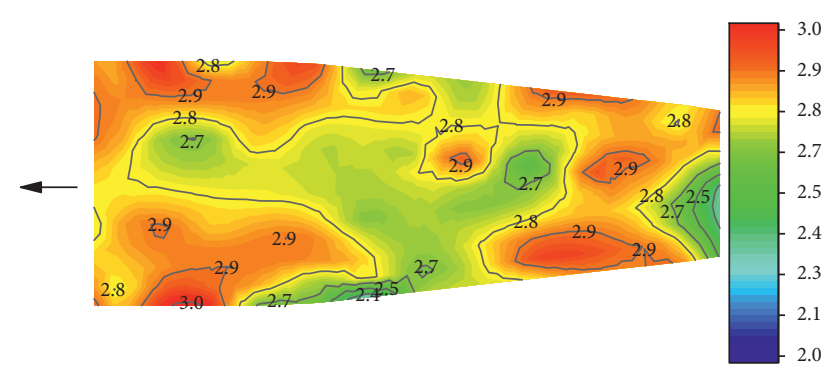

(c)

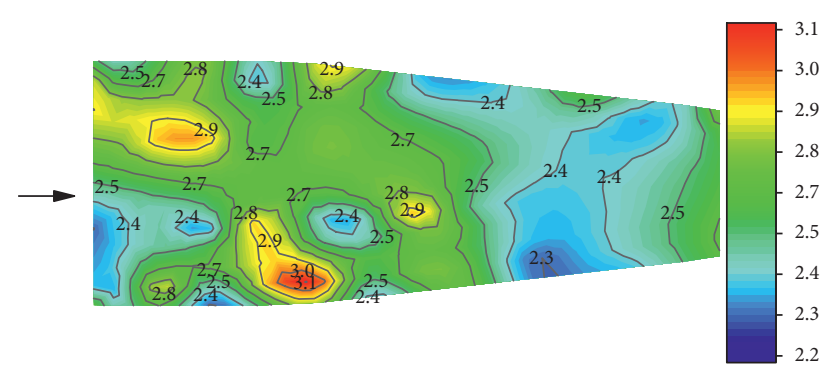

(e)

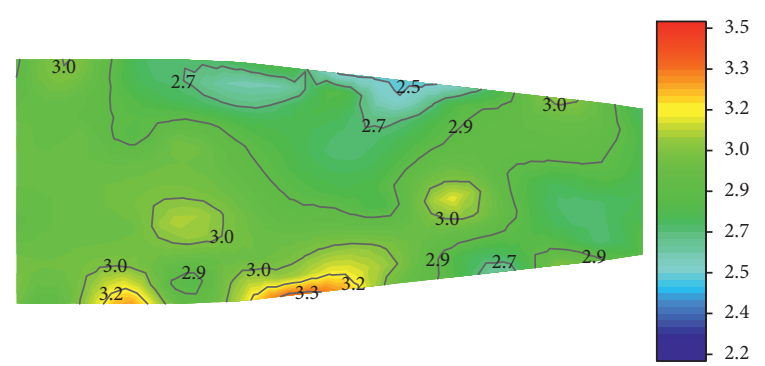

(b)

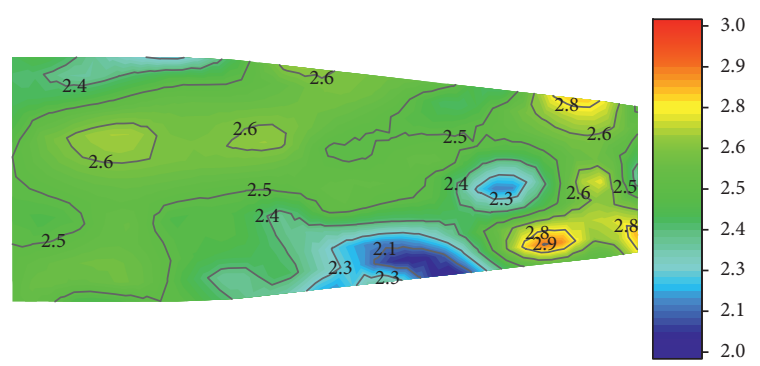

(d)

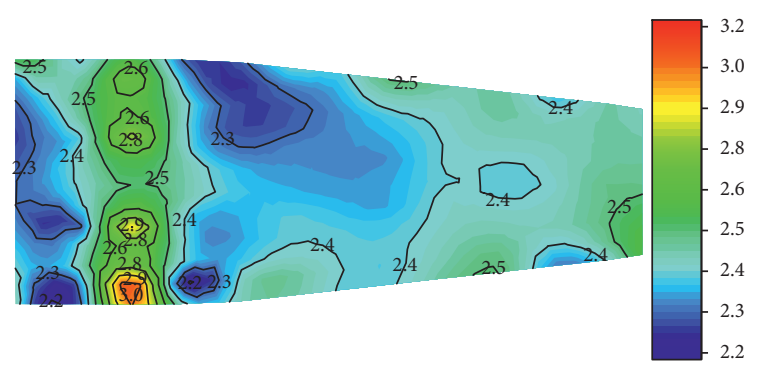

(f)

Figure 17: Peak factors: (a) $g_{\min }-0^{\circ}$, (b) $g_{\max }-0^{\circ}$, (c) $g_{\min }-90^{\circ}$, (d) $g_{\max }-90^{\circ}$, (e) $g_{\min }-270^{\circ}$, and (f) $g_{\max }-270^{\circ}$.

making the favorable wind direction angle of the structure consistent with the regional year-round wind direction.

4.2. New Evaluation Method. In order to obtain the favorable or unfavorable wind direction of the total mean wind pressure coefficient, the corresponding wind pressure coefficient data must first be obtained, which requires a large preorder workload. Based on this, this chapter proposes a method that can quickly judge the favorable or unfavorable wind direction angle only by the shape of the roof.

When the wind flow acts on the windward edge, a section above it will generate vortices due to the separation and reattachment phenomena. Cylindrical vortices tend to occur when the wind flow is perpendicular to the windward edge; otherwise, a conical vortex will form. The area where these vortices are located will generate high negative pressure, which is the main reason for a certain wind direction condition to be an adverse wind direction for the total wind pressure coefficient standard. The roof far from the windward edge usually produces an area that does not fluctuate greatly and maintains a small level wind pressure, and the part outside this area is called the high negative pressure area. The high negative pressure zone is usually closer to the edge of the windward edge and maintains a higher negative pressure level. The data show that the structure of the high negative pressure area is related to the windward edge and wind direction. Wu et al. [23] found that the magnitude of wind speed only affects the wind pressure intensity and does not affect the structure of high negative pressure areas. This chapter borrows the data on the wind pressure distribution of a $600 \mathrm{~mm} \times 600 \mathrm{~mm}$ flat roof from $\mathrm{Li}$ et al. [24] to make the relationship between the high negative pressure area and the wind direction angle more concrete. The structure of the high negative pressure zone at $0^{\circ}$ and $60^{\circ}$ in the original text is simplified, as shown in Figure 19.

The area of the high negative pressure zone is defined as characteristic A. The wind direction angle is counterclockwise rotation, and characteristic A of each part under the wind direction angles of $0^{\circ}$ and $60^{\circ}$ is shown in Table 3 . Assuming that the length of the windward side is $D$, the angle between the wind direction angle and the normal direction of the windward edge is $\alpha$. The mathematical relationship is used to transform each characteristic A including the parameters $D$ and $\alpha$.

It can be concluded that characteristic A under each wind direction angle has a certain geometric relationship with the parameters $D$ and $\alpha$. Ignoring the effect of the error of area 1 , the following formula can be obtained: 


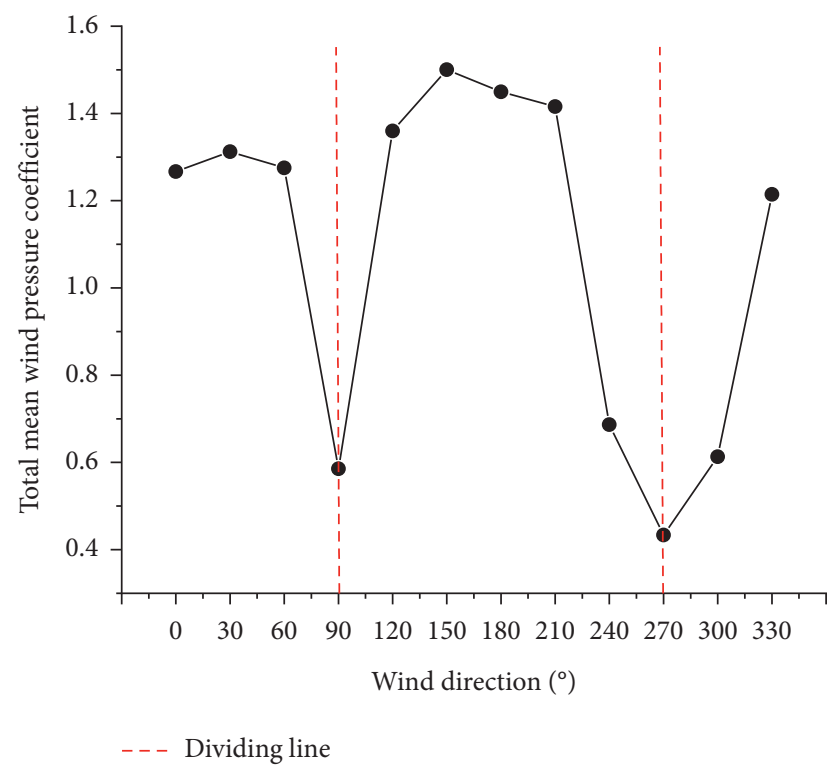

FIGURE 18: Total mean wind pressure coefficient of the whole wind direction.

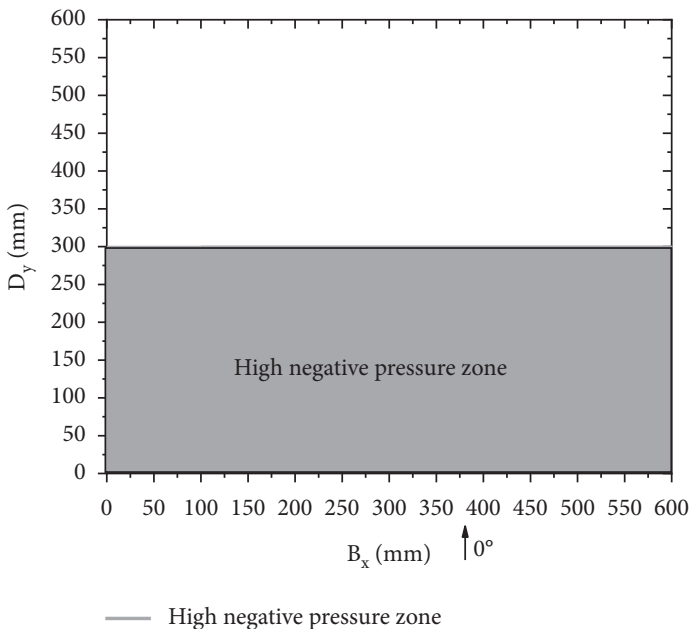

(a)

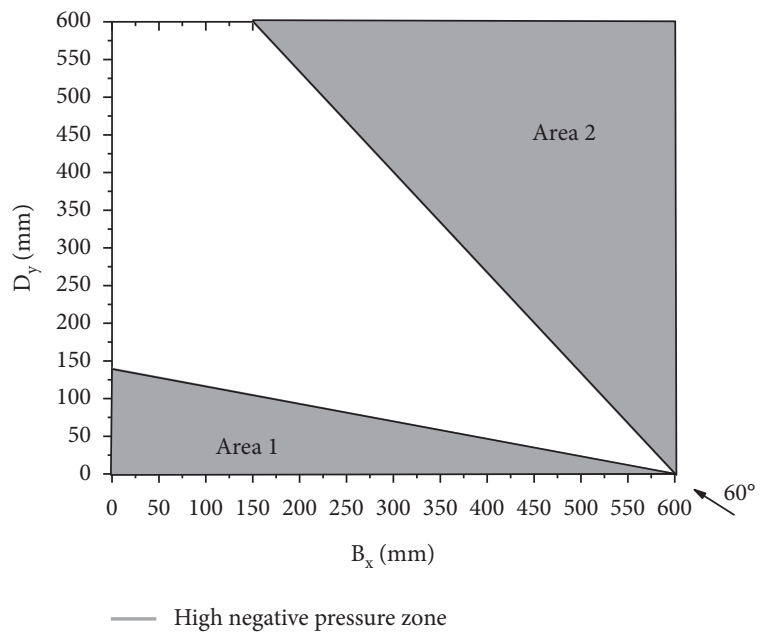

(b)

FIgURE 19: The structure of the high negative pressure zone. (a) For $0^{\circ}$ and (b) for $60^{\circ}$.

TABLE 3: Characteristic and convert.

\begin{tabular}{lccc}
\hline Wind direction & Area & Characteristic A & Convert \\
\hline $0^{\circ}$ & & $600 \times 300=180000$ & $\cos ^{2} 0^{\circ} \times D \times D / 2$ \\
$60^{\circ}$ & 1 & $1 / 2 \times 600 \times 140=42000$ & $\cos ^{2} 60^{\circ} \times D \times(D-40) / 2$ \\
& 2 & $1 / 2 \times 600 \times 450=135000$ & $\cos ^{2} 30^{\circ} \times D \times D / 2$ \\
\hline
\end{tabular}

$$
A=\sum \cos ^{2} \alpha \cdot \frac{D^{2}}{2} .
$$

Meanwhile, A is the sum of the contributions of all windward edges to the structure of high negative pressure area under a certain wind direction angle.

Figure 20 shows the comparison of the trend of the total mean wind pressure coefficient and characteristic $\mathrm{A}$ at each wind direction angle using the roof structure of this paper as an example. To a certain extent, the two trends agree well.
However, the slope and complex shape of regions B and C may affect the accuracy of the method, but they are within the controllable range.

Therefore, for the analysis of favorable and unfavorable wind direction angles based on the total wind pressure coefficient, a rough judgment can be made by comparing the value of characteristic A under each wind direction angle, which will greatly reduce the workload and draw preliminary conclusions only from the shape of the roof. However, this method is suitable for flat roofs or roofs with small arcs. 


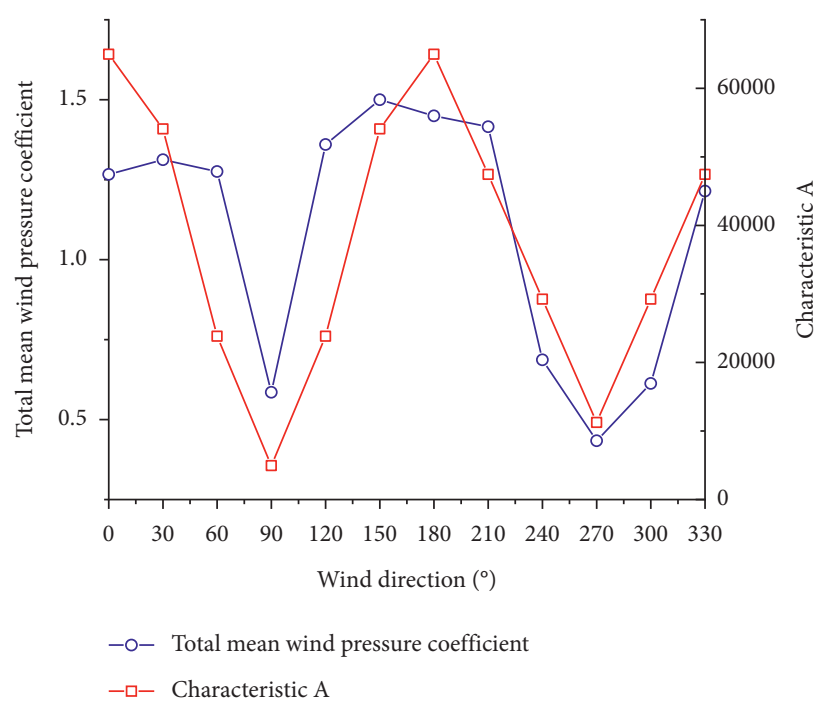

Figure 20: Comparison of the trend of the total mean wind pressure coefficient and characteristic A.

The applicability to larger slopes and more complex shapes remains to be studied. The data cited in the research process of this chapter only include two wind direction angles. Whether more special high negative pressure zone structures will appear under other wind direction angles needs further consideration.

\section{Conclusions}

Based on LES, numerical simulation of the Harbin Wanda Cultural Industry Complex is performed to analyze the wind pressure distribution characteristics of landslide-type roofs. The data of mean wind pressure distribution were compared with the results of existing wind tunnel tests. From the preceding discussion, conclusions are summarized as follows:

(i) The mean wind pressure coefficient predicted by LES and wind tunnel test results show a high similarity. The overall roof shows a high negative pressure, and there is a slight positive pressure at a location remote from the windward edge. For the overall distribution law under a certain wind direction, the maximum value of negative pressure appears near the windward edge and gradually decreases with the distance from the windward edge. When the wind direction angle is perpendicular to the windward edge, a contour line nearly parallel to the windward edge is generated.

(ii) At $0^{\circ}$ wind direction, the maximum negative pressure appears at the junction of area $B$ and area $C$ of the windward edge. The mean wind pressure distributions of $90^{\circ}$ and $270^{\circ}$ show symmetry along the wind direction, and the roof shape is a folding landslide type under these two conditions. At $90^{\circ}$, the height of areas A and B is smaller than that of area $C$, and the wind flow is mostly blocked, resulting in areas $\mathrm{A}$ and $\mathrm{B}$ having a relatively small negative pressure. At $270^{\circ}$, the wind pressures in area $\mathrm{A}$, area $\mathrm{B}$, and area $\mathrm{C}$ are characterized by higher negative pressure, positive pressure, and smaller negative pressure, respectively. Under the full wind direction, the whole roof is subject to a large negative pressure, especially the edge position of the roof, and the negative pressure in the center of areas $\mathrm{A}$ and $\mathrm{C}$ is relatively small.

(iii) For the RMS fluctuating pressures distribution rule, the maximum value appears near the windward edge and decreases with the distance from the windward edge. The value of RMS is related to the elevation and the complexity of the roof surface.

(iv) The non-Gaussian processes distribution of the pressure coefficient is mainly concentrated near the windward edge and roof corners. The maximum peak factor of the roof is 3.5 , which meets the requirements of the whole roof.

(v) Based on the total wind pressure coefficient standard, working conditions except $90^{\circ}, 240^{\circ}, 270^{\circ}$, and $300^{\circ}$ are relatively unfavorable wind directions. A rough method for assessing the unfavorable wind direction is proposed, which only needs to know the shape of the building and the wind direction. However, this method is only suitable for flat roofs and cases where the surface's curvature or slope is relatively slow.

The above conclusions provide a research foundation for the in-depth study of the wind characteristics of landslide-type long-span roof structure.

\section{Data Availability}

The data in this paper are confidential in principle. The raw data can be obtained from the corresponding author upon request.

\section{Conflicts of Interest}

The authors declare that they have no conflicts of interest. 


\section{Acknowledgments}

Wind tunnel test data were provided by China Academy of Building Research and CABR Technology Co., Ltd., which is greatly appreciated.

\section{References}

[1] GB50009-2012, Building Structure Load Specification, China Architecture \& Building Press, Beijing, China, 2012.

[2] O. Nakamura, Y. Tamura, K. Miyashita, and M. Itoh, "A case study of wind pressure and wind-induced vibration of a large span open-type roof," Journal of Wind Engineering and Industrial Aerodynamics, vol. 52, pp. 237-248, 1994.

[3] Y. Uematsu, E. Iizumi, and T. Stathopoulos, "Wind force coefficients for designing free-standing canopy roofs," Journal of Wind Engineering and Industrial Aerodynamics, vol. 95, no. 9-11, pp. 1486-1510, 2007.

[4] C. L. Lu, Q. S. Li, S. H. Huang, F. B. Chen, and X. Y. Fu, "Large eddy simulation of wind effects on a long-span complex roof structure," Journal of Wind Engineering and Industrial Aerodynamics, vol. 100, no. 1, pp. 1-18, 2012.

[5] F. Rizzo and V. Sepe, "Static loads to simulate dynamic effects of wind on hyperbolic paraboloid roofs with square plan," Journal of Wind Engineering and Industrial Aerodynamics, vol. 137, pp. 46-57, 2015.

[6] F. Takeda, T. Yoshino, and Y. Uematsu, "Design wind force coefficients for freestanding canopy roofs of membrane structures," Technical Transactions, vol. 2, no. 12, pp. 167-189, 2015.

[7] K.-S. Kwon, D.-W. Kim, R.-W. Kim, T. Ha, and I.-B. Lee, "Evaluation of wind pressure coefficients of single-span greenhouses built on reclaimed coastal land using a large-sized wind tunnel," Biosystems Engineering, vol. 141, pp. 58-81, 2016.

[8] L. Man, L. Qiu-Sheng, H. Sheng-Hong, S. Feng, and C. Fubin, "Evaluation of wind effects on a large span retractable roof stadium by wind tunnel experiment and numerical simulation," Journal of Wind Engineering and Industrial Aerodynamics, vol. 179, pp. 39-57, 2018.

[9] X. Sun, R. Yu, and Y. Wu, "Investigation on wind tunnel experiments of ridge-valley tensile membrane structures," Engineering Structures, vol. 187, pp. 280-298, 2019.

[10] J. Colliers, M. Mollaert, J. Degroote, and L. De Laet, "Prototyping of thin shell wind tunnel models to facilitate experimental wind load analysis on curved canopy structures," Journal of Wind Engineering and Industrial Aerodynamics, vol. 188, pp. 308-322, 2019.

[11] Z. Chen, H. Li, X. Wang, X. Yu, and Z. Xie, "Internal and external pressure and its non-Gaussian characteristics of long-span thin-walled domes," Thin-Walled Structures, vol. 134, pp. 428-441, 2019.

[12] F. Rizzo, V. Sepe, F. Ricciardelli, and A. M. Avossa, "Wind pressures on a large span canopy roof," Wind and Structures, vol. 30, no. 3, pp. 299-316, 2020.

[13] B. Blocken, "50 years of computational wind engineering: past, present and future," Journal of Wind Engineering and Industrial Aerodynamics, vol. 129, pp. 69-102, 2014.

[14] S. H. Huang, Q. S. Li, and J. R. Wu, "A general inflow turbulence generator for large eddy simulation," Journal of Wind Engineering \& Industrial Aerodynamics, vol. 98, no. 10-11, pp. 600-617, 2010.

[15] S. J. Daniels, I. P. Castro, and Z.-T. Xie, "Peak loading and surface pressure fluctuations of a tall model building," Journal of Wind Engineering and Industrial Aerodynamics, vol. 120, pp. 19-28, 2013.

[16] N. S. Fouad, G. H. Mahmoud, and N. E. Nasr, "Comparative study of international codes wind loads and CFD results for low rise buildings," Alexandria Engineering Journal, vol. 57, no. 4, pp. 3623-3639, 2018.

[17] K. Wijesooriya, D. Mohotti, K. Chauhan, and D. Dias-da-Costa, "Numerical investigation of scale resolved turbulence models (LES, ELES and DDES) in the assessment of wind effects on supertall structures," Journal of Building Engineering, vol. 25, 2019.

[18] J. Franke, A. Hellsten, K. H. Schlunzen, and B. Carissimo, "The COST 732 best Practice Guideline for CFD simulation of flows in the urban environment: a summary," International Journal of Environment and Pollution, vol. 44, no. 1/2/3/4, pp. 419-427, 2011.

[19] B. W. Yan and Q. S. Li, "Inflow turbulence generation methods with large eddy simulation for wind effects on tall buildings," Computers \& Fluids, vol. 116, pp. 158-175, 2015.

[20] A. Smirnov, S. Shi, and I. Celik, "Random flow generation technique for large eddy simulations and particle-dynamics modeling," Journal of Fluids Engineering, vol. 123, no. 2, p. $359,2001$.

[21] ISO4354:2009(E), Wind Actions on Structures, International Organization for Standardization, Geneva, Switzerland, 2nd edition, 2009.

[22] K. S. Kumar and T. Stathopoulos, "Wind loads on low building roofs: a stochastic perspective," Journal of Structural Engineering, vol. 126, no. 8, pp. 944-956, 2000.

[23] F. Wu, P. P. Sarkar, K. C. Mehta, and Z. Zhao, "Influence of incident wind turbulence on pressure fluctuations near flat-roof corners," Journal of Wind Engineering and Industrial Aerodynamics, vol. 89, no. 5, pp. 403-420, 2001.

[24] B. Li, Y. Tian, Q. Yang, and Z. Fan, "Research on design wind loads on cladding and components of flat roof," Journal of Building Structures, vol. 37, 2016. 